\title{
MÔ HÌNH ĐÁNH GIÁ CHẤT LƯợNG BẢN DỊCH VÀ ĐỀ XUẤT ĐỐI VỚI ĐÁNH GIÁ DỊCH ANH - VIẸT
}

\author{
Triệu Thu Hằng* \\ Khoa Su phạm tiếng Anh, Trường Đại học Ngoại ngũ, ĐHQGHN, \\ Phạm Văn Đồng, Cầu Giá́y, Hà Nội, Việt Nam \\ Nhận bài ngày 11 tháng 08 năm 2017 \\ Chỉnh sửa ngày 05 tháng 09 năm 2017; Chấp nhận đăng ngày 28 tháng 09 năm 2017
}

Tóm tắt: Bài báo điểm lại các hướng tiếp cận trong đánh giá dịch thuật, trong đó có một số hướng tiếp cận chính là tiền ngôn ngữ, đánh giá bản dịch dựa trên phản hồi của độc giả và đánh giá bản dịch dựa trên phân tích văn bản và diễn ngôn. Để phục vụ cho thực tiễn đánh giá, chúng tôi lựa chọn mô tả, phân tích và bàn luận mô hình chức năng-dụng học của House (2015) thuộc hướng tiếp cận đánh giá bản dịch dựa trên phân tích văn bản và diễn ngôn. Trên cơ sở đó, chúng tôi đưa ra những gợi ý và đề xuất đối với đánh giá dịch thuật Anh-Việt ${ }^{1}$.

Tù khoá: đánh giá chất lượng bản dịch, phản hồi độc giả, phân tích văn bản và diễn ngôn, mô hình của House (2015)

\section{Dẫn nhập}

Nhiều năm qua, lĩnh vực đánh giá dịch thuật luôn thu hút các học giả trong nước và quốc tế (House, 1997; Nord, 1997; Lauscher, 2000; Brunette, 2000; Colina, 2008; William, 2009). Những nghiên cứu trước đây (Wilss, 1996; Schäffer, 1998; Al-Quinai, 2000; Moskal, 2000; Melis \& Hurtado, 2001; Mossop, 2007; Williams, 2009; Manfredi, 2012; Butler \& MeMunn, 2014; Colina, 2015) chỉ ra rằng việc đánh giá không chính xác chất lượng bản dịch có thể ảnh hưởng tiêu cực đến chất lượng bản dịch và người dịch. Xuất phát từ tầm quan trọng của đánh giá dịch thuật, bài báo này điểm lại các hướng tiếp cận chính trong đánh giá dịch thuật, bao gồm tiền ngôn ngữ, đánh giá bản dịch dựa trên phản hồi của độc giả và đánh giá bản dịch dựa trên phân tích văn bản và diễn ngôn. Để phục vụ cho thực tiễn đánh giá, chúng tôi lựa chọn mô tả, phân tích và bàn luận mô hình của House

\footnotetext{
* ĐT.: 84-944811991

Email: trieuthuhang91@gmail.com

${ }^{1}$ Bài báo này được hoàn thành với sự hỗ trợ của Trường Đại học Ngoại ngữ - Đại học Quốc gia Hà Nội trong đề tài mã số QG.15.35 "Nghiên cứu mô hình đánh giá dịch thuật Anh-Việt".
}

(2015) thuộc hướng tiếp cận đánh giá bản dịch dựa trên phân tích văn bản và diễn ngôn. Trên cơ sở đó, chúng tôi đưa ra những gợi ý và đề xuất đối với đánh giá dịch thuật Anh-Việt.

\section{Các hướng tiếp cận trong đánh giá dịch thuật}

\subsection{Tiền ngôn ngũu (Mentalist views)}

Ở thời kì tiền ngôn ngữ, các tiêu chí đánh giá tương đối chung chung và phụ thuộc vào yếu tố cảm tính cũng như trực giác của người đánh giá. Ví dụ, ba tiêu chuẩn "Tín-ĐạtNhã” của Nghiêm Phục tại Trung Quốc cho thấy thiếu tính khả thi và dựa trên cảm tính của người đánh giá. Còn trong lý luận dịch phương Tây không thể không kể đến đại luận của Tytler vào thế kỷ 18 , ông cho rằng một bản dịch tốt cần hội tụ những yếu tố quan trọng bậc nhất là "tinh hoa của nguyên tác được chuyển hoàn toàn qua ngôn ngữ đích sao cho người bản xứ đọc bản dịch ra tiếng nước mình thấu hiểu và cảm nhận mạnh mẽ giống như cách hiểu và cảm nhận của người nói ngôn ngữ của nguyên tác" (phần dịch của Hồ Đắc Túc, 2012:61). Tuy nhiên, làm thế nào để đánh giá được độc giả ngôn ngữ đích cảm nhận bản dịch giống như cảm nhận của người 
nói ngôn ngữ của nguyên tác vẫn là một vấn đề ngỏ.

\section{2. Đánh giá bản dịch dụa trên phản hồi của độc giả (Response-based approach)}

Các học giả ủng hộ hướng tiếp cận đánh giá bản dịch dựa trên phản hồi của độc giả (Nida, 1964; Nida \& Taber, 1969; Reiß \& Vermeer, 1984; Holz-Mänttäri, 1986; Nord, 1991) cho rằng đánh giá chất lượng của một bản dịch cần dựa trên phản hồi của đối tượng độc giả ở ngôn ngữ đích (đối tượng tiếp nhận bản dịch). Nói cách khác, chất lượng bản dịch cần được đánh giá dựa trên thành công của bản dịch trong đời sống thực tế của nó. Xuất phát từ quan điểm này, có hai hướng tiếp cận như sau.

\subsubsection{Ngôn ngũ tâm lý học trong đánh giá} bản dịch (Behavioristic views)

Kể từ thập niên 1960, chịu ảnh hưởng của hành vi luận tại Mỹ, Nida (1964) đã đề xuất một số bài kiểm tra phản hồi của độc giả để đưa ra kết luận về chất lượng của bản dịch. Nida đưa ra một số dạng bài để kiểm tra phản hồi của độc giả như như đọc văn bản thành tiếng (reading aloud techniques), bài điền từ (cloze task) và đánh giá bản dịch theo các mức độ (rating tasks). Phát triển theo quan điểm của Nida (1964), Nida \& Taber (1969:169-172) đề xuất đánh giá bản dịch dựa vào phản ứng độc giả thông qua ba yếu tố: độc giả ngữ đích hiểu thông điệp của bản gốc đúng đến mức độ nào, bản dịch có dễ hiểu không, và sự tham gia trải nghiệm của cá nhân độc giả. Tuy nhiên, những bài kiểm tra này chưa được áp dụng rộng rãi vì các bài kiểm tra dựa trên những phản hồi cảm tính độc giả chưa đủ để phản ánh toàn diện vấn đề chất lượng bản dịch (House, 2015). Thêm vào đó, có thể thấy hạn chế của hướng tiếp cận này là vai trò của bản gốc không được xét đến trong quá trình đánh giá.

\subsubsection{Trường phái chức năng của Đức} (Functionalist approach)

Trường phái chức năng của Đức còn được gọi là trường phái Skopos. Thuật ngữ "Skopos" bắt nguồn từ tiếng Hy Lạp, có nghĩa là "mục đích". Theo lý thuyết Skopos khởi xướng bởi hai học giả người Đức Reiß \& Vermeer (1984), dịch thuật là một hành động có mục đích và yếu tố quan trọng bậc nhất trong toàn bộ quá trình dịch là mục đích của bản dịch. Trong quá trình đánh giá bản dịch cũng vậy, tiêu chí quan trọng bậc nhất để đánh giá bản dịch là bản dịch có đạt được mục đích hay không. Một yếu tố quan trọng cần xét tới để đạt được mục đích của bản dịch là độc giả (đối tượng tiếp nhận bản dịch ở ngôn ngữ đích). Độc giả ở ngôn ngữ đích có kiến thức văn hoá riêng, những kì vọng riêng và nhu cầu giao tiếp riêng của họ; và mục đích của bản dịch là làm sao để đáp ứng nhu cầu của nhóm đối tượng độc giả cụ thể.

Những nguyên tắc theo lý thuyết Skopos được mô tả theo trình tự như sau: (1) Bản dịch được quyết định bởi mục đích dịch; (2) Bản dịch là một phương án về thông điệp trong ngôn ngữ và văn hoá đích dựa trên phương án về thông điệp trong ngôn ngữ và văn hoá nguồn; (3) Phương án về thông điệp trong ngôn ngữ và văn hoá đích và phương án về thông điệp trong ngôn ngữ và văn hoá nguồn không phải là chỉ có một phương án duy nhất; (4) Bản dịch cần đảm bảo được tính mạch lạc (đáp ứng nhu cầu độc giả ở ngữ đích); (5) Bản dịch cần đảm bảo tính mạch lạc, gắn kết với văn bản nguồn; (6) Năm nguyên tắc trên được sắp đặt theo trình tự, trong đó nguyên tắc đầu tiên quan trọng bậc nhất.

Ngoài ra, Reiß (1973, dựa vào bản dịch của Rhodes 2014) chỉ ra rằng cần xác định loại văn bản (text type) trong quá trình chất lượng bản dịch. Mỗi thể loại văn bản có những yêu cầu, đặc điểm riêng trong quá trình dịch cũng như đánh giá. Bốn thể loại văn bản chính Reiß (1973) đưa ra là văn bản hướng nội dung (ví dụ: tin tức, văn bản khoa học); văn bản hướng hình thức (ví dụ: thơ ca, văn học); văn bản thể hiện ý muốn (ví dụ: quảng cáo), và văn bản dạng âm thanh, truyền thông (ví dụ: kịch nói). 
Điểm mạnh của lý thuyết Skopos là một bản gốc có thể được dịch theo nhiều sách lược, phương án khác nhau dựa trên những mục đích khác nhau của bản dịch. Ngoài ra, người dịch được trao quyền tự do, có một "dư địa rộng lớn” (Lê Hoài Ân, 2017) trong việc lựa chọn những chiến lược, phương pháp dịch để đạt được những mục đích dịch khác nhau mà người dịch đang nhằm hướng tới. Tuy nhiên, đồng quan điểm với Nord (1997:109-122) và Schäffer (1997:237-238), chúng tôi cho rằng thuyết Skopos không phù hợp đối với đánh giá dịch một số thể loại văn bản nhất định, cụ thể là đánh giá dịch văn học vì thuyết Skopos không coi trọng bản gốc và những đặc điểm ngôn ngữ của bản gốc, mà trong văn học, đặc biệt là những kiệt tác có giá trị nghệ thuật, đặc điểm ngôn ngữ là một yếu tố không thể bỏ qua.

\section{3. Đánh giá dịch thuật dụa trên phân tích} văn bản và diễn ngôn (Text and Discoursebased approach)

\subsection{1. Đánh giá chỉ dụa trên bản dịch}

Tác giả tiêu biểu của hướng tiếp cận này là Toury (1995) trong đó ông cho rằng chất lượng bản dịch được thể hiện thông qua hình thức và chức năng bên trong hệ thống văn bản và văn hoá đích. Khác với hướng tiếp cận so sánh đối chiếu (Steiner, 1998; Nord, 2005; House, 2015), các tiêu chí Toury đưa ra chỉ tập trung phân tích, đánh giá bản dịch, và hệ thống ngôn ngữ đích. Vì vậy, hướng tiếp cận này có phần chưa phù hợp vì đánh giá dịch thuật cần xem xét kĩ lưỡng cả bản gốc và bản dịch (Phạm Thị Thuỷ, 2013).

\subsubsection{Chủ nghĩa hậu hiện đại và giải kiến} tạo (Post-modernist and Deconstructionist Thinking)

Các tác giả của của chủ nghĩa hậu hiện đại và giải kiến tạo (Derrida, 1985; Graham, 1995; deMan 1986; Benjamin, 1989; Venuti, 1995; Gentzler, 1993; trích trong House, 1977) nghiên cứu thực tiễn dịch thuật từ góc độ triết học, tâm lý, và chính trị, xã hội để giải quyết vấn đề bất bình đẳng. Hướng tiếp cận này tập trung sâu vào những yếu tố ẩn hình trong quá trình dịch như những thay đổi, chỉnh sửa trong bản gốc để phục vụ lợi ích của những cá nhân và nhóm người nắm quyền lực, việc lựa chọn các văn bản nguồn để dịch và các chiến lược, phương pháp dịch được áp dụng. Nhận định về hướng tiếp cận này, chúng tôi đồng ý với quan điểm của House (2001) rằng việc so sánh đối chiếu giữa bản gốc và bản dịch không chỉ dừng ở vấn đề hệ tư tưởng (ideology) mà cần nghiên cứu nhiều yếu tố khác nữa.

\subsubsection{Huớng tiếp cận dự trên ngôn ngư} hoc (Linguistics-based approaches)

Hướng tiếp cận dịch thuật dựa trên ngôn ngữ phát triển từ thập niên 1950 với đóng góp tiên phong của Catford (1965) với nỗ lực thực hiện đối chiếu ngôn ngữ (contrastive linguistics) trong nghiên cứu dịch thuật. Nhiều học giả khác tiếp nối quan điểm của Catford (1965) hướng đến nghiên cứu dịch thuật dựa trên quan điểm ngôn ngữ học như Baker (1992), Hatim \& Mason (1997), House (1997, 2015), Hickey (1998), Steiner (1998), Hatim \& Munday (2004), Teich (2004) và Munday (2008). Theo hướng tiếp cận này, cả bản gốc và bản dịch đều đóng vai trò quan trọng trong quá trình đánh giá bản dịch. Mô hình chức năng-dụng học của House (1977, 1997, 2015) là một trong những mô hình theo hướng tiếp cận đánh giá bản dịch dựa trên phân tích văn bản, và đã được thử nghiệm áp dụng để đánh giá bản dịch ở nhiều cặp ngôn ngữ khác nhau (Jiang, 2010; Nazhand \& Mohebbi Pur, 2011; Alikademi, 2015; Tabrizi, Chalak \& Taheroun 2013; Ehsani \& Zohrabi, 2014; Hassan, 2015; Rahmanian, 2015; Zekri \& Shahsavar, 2016).

Nhằm đóng góp cho cơ sở lý luận xây dựng mô hình đánh giá dịch của cặp ngôn ngữ Anh-Việt, bài báo này lựa chọn bàn luận mô hình của House (2015). Có thể thấy, nhiều tác giả đã nỗ lực xây dựng các mô hình đánh giá dịch thuật như mô hình của House (1977, 
1997, 2015), Newmark (1988), Hatim và Mason (1990, 1997), Baker (1992), Steiner (1998), Waddington (2001), Williams (2009), Nord (1991, 1997, 2005), Al-Qinai (2000). Để phục vụ cho thực tiễn đánh giá dịch Anh-Việt, mô hình của House (2015) với những thao tác vận hành chi tiết dựa trên cơ sở lý thuyết cụ thể của Crystal \& Davy (1969), Halliday (1973), được chúng tôi lựa chọn để mô tả và đánh giá trong phần tiếp theo dưới đây.

\section{Mô hình chức năng-dụng học của House (2015)}

Juliane House là một nhà nghiên cứu dịch thuật người Đức, bà là Chủ tịch Hiệp hội quốc tế về nghiên cứu dịch và liên văn hoá (International Association for Translation and Intercultural Studies). Mô hình chức năngdụng học của House $(1977,1997,2015)$ dựa một phần trên nền tảng lý thuyết ngôn ngữ học chức năng hệ thống (Halliday, 1973) và ngữ dụng (Austin, 1962; Searle, 1972).

Tương đồng với quan điểm của các học giả ủng hộ quan điểm nghiên cứu dịch cần dựa trên nền tảng ngôn ngữ, House (1997:31) đề cao vai trò của phân tích văn bản trong đánh giá chất lượng bản dịch. Để thực hiện phân tích văn bản, House cho rằng cần đặt văn bản vào hoàn cảnh, tình huống cụ thể. Mô hình chức năng-dụng học của House được xây dựng dựa trên hai khái niệm "ngôn cảnh tình huống" và "ngôn cảnh văn hoá" của nhà nhân chủng học Malinowski (1923) khi ông làm việc thực tế giữa hai nền văn hoá xa lạ và dựa trên mô hình phân tích văn bản của Crystal \& Davy (1969). Hai khái niệm này đóng vai trò quan trọng trong việc giúp người dịch cần phải không chỉ phân tích ngôn bản, nắm bắt được những gì đang xảy ra mà còn cần nắm bắt được cả nền văn hoá tổng thể, hiểu được một cách đầy đủ các ý nghĩa của ngôn bản, quan điểm này được các nhà nghiên cứu ủng hộ rộng rãi như Firth (1957), Hymes (1964, 1974), Halliday (1973), Halliday \& Hasan (1989), Martin \& Rose (2008).
Việc phân tích văn bản trong ngôn cảnh tình huống cụ thể được hiện thực hoá thông qua phân tích Ngữ vực, bao gồm Trường (Field), Không khí của ngôn bản (Tenor) và Phương thức giao tiếp của ngôn bản (Mode). Ngoài ra, ngôn cảnh văn hoá được thể hiện qua phân tích Thể loại văn bản (Genre). Mô hình của House được trình bày ở Hình 1 dưới đây:

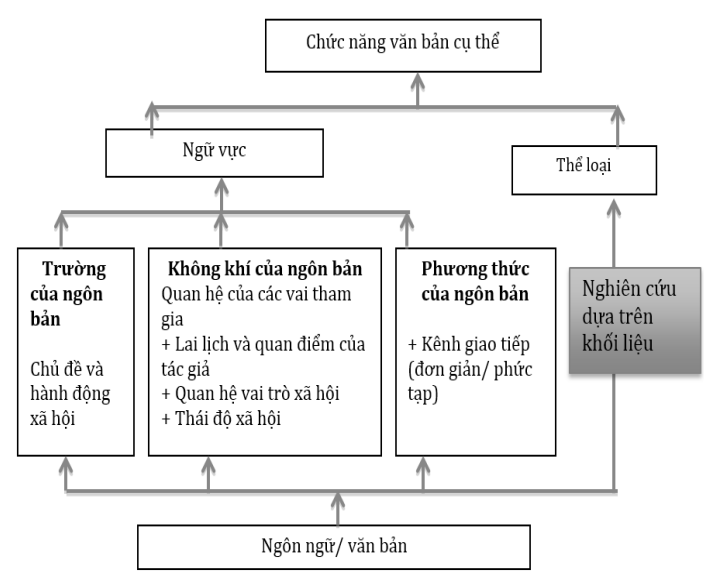

Hình 1. Mô hình chức năng-dụng học của House (2015: 127)

House đã liên tục chỉnh sửa và cập nhật mô hình chức năng dụng học trên qua các năm 1977, 1997, 2015 dựa trên nghiên cứu thực nghiệm. Dựa trên quan điểm lý thuyết chức năng hệ thống của Halliday (1973), khái niệm "chức năng" trong mô hình này được hiểu là chức năng của văn bản "việc sử dụng văn bản trong một tình huống cụ thể" (Lyons, 1969:434). Để xác định được chức năng của văn bản theo quan điểm này, người đánh giá cần phân tích văn bản đặt trong ngôn cảnh tình huống và ngôn cảnh văn hoá thông qua phân tích Ngữ vực và Thể loại. House (2015) có phân biệt cụ thể "chức năng ngôn ngữ” và “chức năng văn bản" là hai khái niệm khác nhau. Như đã đề cập, chức năng văn bản "việc sử dụng văn bản trong một tình huống cụ thể" (Lyons, 1969:434). Về chức năng ngôn ngữ, House (2015) cho rằng ngôn ngữ có hai chức năng, đó là chức năng ý niệm và chức năng liên nhân. 
Về các thuật ngữ, "Trường của ngôn bản" bao quát chủ đề và hoạt động xã hội. "Không khí của ngôn bản" chỉ mối quan hệ giữa những người tham gia giao tiếp về quyền lực xã hội, khoảng cách xã hội, lai lịch và quan điểm của tác giả, thái độ xã hội. "Phương tiện của ngôn bản" chỉ phương tiện, kênh giao tiếp của ngôn bản (nói hoặc viết).

Để rút ra kết luận về chức năng văn bản, thuật ngữ "Thể loại" (Genre) được House đưa vào mô hình. Trong mô hình cập nhật của House (2015), bà nhấn mạnh tầm quan trọng của các kết quả từ nghiên cứu dựa trên khối liệu (corpus studies) để xác định thể loại văn bản. Theo Derewianka (1990: 18), thuật ngũ̃ "Thể loại" (genre) được dùng để chỉ một loại văn bản cụ thể. Thể loại của văn bản được xác định một phần bởi nền văn hoá mà ở đó văn bản được sử dụng, bởi vì những nền văn hoá khác nhau có cách sử dụng ngôn ngữ, cấu trúc văn bản khác nhau để đạt được mục đích. Hình 2 dưới đây thể hiện mối quan hệ giữa Văn bản, Ngữ vực và Thể loại.

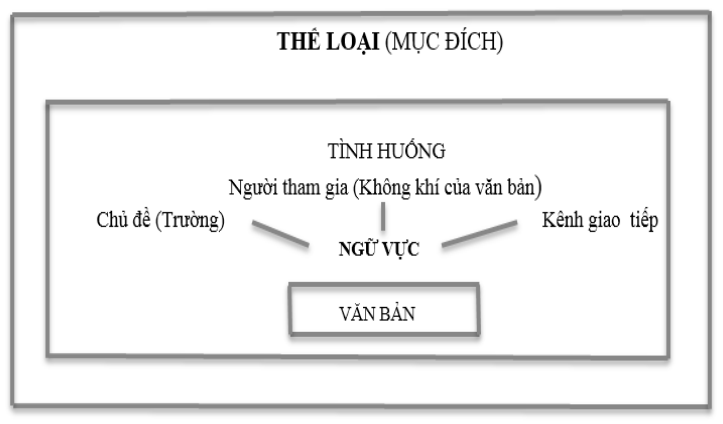

Hình 2. Mối quan hệ giữa Văn bản, Ngữ vực và Thể loại dựa theo Derewianka (1990:19)

Việc phân tích văn bản đặt trong ngôn cảnh tình huống và ngôn cảnh văn hoá thông qua phân tích Ngữ vực và Thể loại giúp kết luận về chức năng của văn bản. House (1981:49) cho rằng văn bản dịch không chỉ phù hợp với văn bản gốc về chức năng, mà cần sử dụng các phương tiện từ vựng, cú pháp và liên kết văn bản để đạt được chức năng đó.
Mô hình của House được thực hiện qua 6 thao tác như sau: (1) phân tích bản gốc để lập hồ sơ bản gốc theo Ngữ vực (Trường, Không khí ngôn bản, Phương thức của ngôn bản), việc phân tích bản gốc này được đặt vào ngôn cảnh tình huống thông qua phân tích các phương tiện từ vựng, cú pháp và liên kết văn bản; (2) mô tả thể loại của bản gốc; (3) kêt luận về chức năng của bản gốc, bao gồm chức năng ý niệm và chức năng liên nhân; (4) phân tích hồ sơ bản dịch và chức năng bản dịch tương tự như các bước thực hiện ở bản gốc; (5) so sánh hồ sơ bản dịch và hồ sơ bản gốc và đưa ra kết luận về những vấn đề trong bản dịch, những lỗi dịch so với bản gốc; (6) kết luận về chất lượng của bản dịch.

Mô hình của House (2015) có những điểm mạnh và hạn chế riêng. Trước hết, mô hình này có thể áp dụng để đánh giá nhiều thể loại văn bản khác nhau. Các nghiên cứu trên thế giới (Jiang, 2010; Nazhand \& MohebbiPur, 2011; Alikademi, 2015; Tabrizi, Chalak \& Taheroun, 2013; Ehsani \& Zohrabi, 2014; Hassan, 2015; Rahmanian, 2015; Zekri \& Shahsavar, 2016; Kargarzadeh \& Paziresh, 2017) áp dụng mô hình chức năng-dụng học của House trong đánh giá dịch văn học, thơ, văn bản quảng cáo, y học, thuật ngữ tôn giáo, văn bản quảng cáo du lịch, các chỉ dẫn ở viện bảo tàng, v.v. Ở Việt Nam, một số luận văn, luận án tại Trường Đại học Ngoại ngữ - Đại học Quốc gia Hà Nội áp dụng mô hình chức năng-dụng học của House (1977, 1997) trong đánh giá dịch văn học Anh-Việt như Lê Mỹ Hạnh (2009), Đặng Thị Phượng (2013), Cao Huyền Trang (2014), Triệu Thu Hằng (2015), Phạm Thị Thuỷ (2015) và kêt quả nghiên cứu từ các luận văn, luận án này bước đầu cho thấy mô hình của House (1997) có khả năng áp dụng cao trong đánh giá dịch văn học Anh-Việt. Ngoài ra, Steiner (1998:17) cho rằng mô hình của House kêt hợp tốt giữa cấp độ phân tích các đặc điểm từ vựng ngữ pháp và cấp độ cao hơn là cấp 
độ ngữ vực và thể loại để đưa ra kết luận về chức năng văn bản. Hơn nữa, House không chỉ xét đến phương diện văn bản đặt trong tình huống, mà phương diện văn hoá của văn bản cũng được nghiên cứu, đây là lợi thế cho việc đánh giá văn bản ở những cặp ngôn ngữ thuộc những nền văn hoá khác nhau, ví dụ đánh giá dịch Anh-Việt. Thêm vào đó, các tác giả như Steiner (1998) và Phạm Thị Thuỷ (2013) cho rằng mô hình của House dựa trên nền tảng, cơ sở ngôn ngữ học rõ ràng với các thao tác áp dụng mô hình cụ thể, chi tiết, đóng góp cho cả nghiên cứu dịch và nghiên cứu ngôn ngữ.

Tuy nhiên, Gutt (1991:46-49) cho rằng mô hình của House (1977) giúp người đánh giá tìm ra những điểm không tương đương về chức năng (mismatches) giữa bản gốc và bản dịch. Trong khi những điểm không tương đương về chức năng (mismatches) có thể là những lỗi dịch, nhưng cũng có thể do sự khác biệt về ngôn ngữ và văn hoá. Venuti (1995:21) cũng tranh luận rằng sự khác biệt về ngôn ngữ cho thấy sự khác biệt về văn hoá. Theo quan điểm của Venuti (1995:21), tiếp cận dịch theo hướng trên có phần tạo ra một mô hình cứng nhắc, giới hạn vai trò của dịch trong xu hướng đổi mới văn hoá. Để khắc phục vấn đề này, kể từ năm 1997 cho tới 2015, House thực hiện dụng học đối chiếu (contrastive pragmatics) trong dự án Hamburg "Dịch hướng đích" (Covert translation) trong đó có đưa ra khái niệm "bộ lọc văn hoá" (cultural filter) để giải quyết sự khác biệt về văn hoá xã hội giữa những cặp ngôn ngữ khác nhau. Tóm lại, những điểm chính về mô hình chức năng-dụng học của House được thể hiện qua Bảng 1. Phân tích của chúng tôi về mô hình của House (2015) như sau:

\begin{tabular}{|c|c|}
\hline & $\begin{array}{c}\text { Mô hình của House }(1977, \text { 1997, } \\
\text { 2015) }\end{array}$ \\
\hline $\begin{array}{l}\text { Quan } \\
\text { điểm } \\
\text { chính }\end{array}$ & $\begin{array}{l}\text { Bản dịch cần đạt được chức năng } \\
\text { tương đương so với chức năng bản } \\
\text { gốc (chức năng ở đây được hiểu là } \\
\text { chức năng của văn bản. Chức năng } \\
\text { của văn bản được xác định bởi phân } \\
\text { tích văn bản đật trong ngôn cảnh tình } \\
\text { huống và ngôn cảnh văn hoá thông } \\
\text { qua Ngữ vực và Thể loại). }\end{array}$ \\
\hline $\begin{array}{l}\text { Nền tảng } \\
\text { lý thuyết }\end{array}$ & $\begin{array}{l}\text { - Đối chiếu ngôn ngữ (Contrastive } \\
\text { linguistics) } \\
\text { - Ngôn ngữ học chức năng hệ thống } \\
\text { (Systemic Functional Theory) } \\
\text { - Lý thuyết Ngữ vực (Register } \\
\text { Theory) } \\
\text { - Nghiên cứu dựa trên khối liệu } \\
\text { (Corpus Studies) }\end{array}$ \\
\hline $\begin{array}{l}\text { Các thao } \\
\text { tác áp } \\
\text { dụng }\end{array}$ & $\begin{array}{l}\text { - Phân tích bản gốc và xác định chức } \\
\text { năng của bản gốc; } \\
\text { - Phân tích bản dịch và xác định chức } \\
\text { năng của bản dịch; } \\
\text { - So sánh hồ sơ bản gốc và bản dịch; } \\
\text { - Phân loại lỗi dịch (lỗi dịch hướng } \\
\text { nguồn, lồi dịch hướng đích); } \\
\text { - Nhận xét và kết luận về chất lượng } \\
\text { bản dịch. }\end{array}$ \\
\hline $\begin{array}{l}\text { Các tiêu } \\
\text { chí phân } \\
\text { tích văn } \\
\text { bản }\end{array}$ & $\begin{array}{l}\text { - Phân tích Ngữ vực (Trường, Không } \\
\text { khí ngôn bản, Phương thức ngôn bản) } \\
\text { và Thể loại thông qua phương tiện từ } \\
\text { vựng, cú pháp và liên kêt văn bản. }\end{array}$ \\
\hline $\begin{array}{l}\text { Điểm } \\
\text { mạnh }\end{array}$ & $\begin{array}{l}\text { - Phân tích văn bản một cách đầy đủ } \\
\text { bao gồm các yếu tố ngoại ngôn và nội } \\
\text { ngôn (thể hiện qua phân tích Ngữ vực } \\
\text { và thể loại); } \\
\text { - Có thế áp dụng với nhiều thể loại văn } \\
\text { bản khác nhau (đã qua thử nghiệm); } \\
\text { - Kết hợp phương pháp nghiên cứu } \\
\text { định tính (phương pháp chính) và } \\
\text { định lượng (phương pháp phụ) trong } \\
\text { quá trình đánh giá. }\end{array}$ \\
\hline Hạn chế & $\begin{array}{l}\text { - Thuật ngữ không tương đương về } \\
\text { chức năng (mismatches) và lồi dịch } \\
\text { (translation errors) cần được phân } \\
\text { biệt rõ ràng. }\end{array}$ \\
\hline
\end{tabular}

Từ phần bàn luận về hướng tiếp cận đánh giá bản dịch dựa trên phản hồi của độc giả và hướng tiếp cận đánh giá bản dịch dựa trên phân tích văn bản và diễn ngôn cũng như mô hình của House (2015), chúng tôi đưa ra một 
số nhận xét như sau. Có thể thấy, mô hình của House (2015) đặt trọng tâm vào phân tích văn bản, cả văn bản nguồn và văn bản dịch. Chúng tôi đồng ý với quan điểm của House (1977, 1997, 2015) về vai trò của phân tích văn bản nguồn và văn bản dịch, và người đánh giá cần thực hiện những mô tả về ngôn ngữ trước khi đi đến dựa trên những đánh giá mang tính trực giác từ phía độc giả. House (2001) biện luận rằng mặc dù đánh giá bản dịch cần xét tới yếu tố xã hội như người dịch tiếp nhận bản dịch như thế nào, v.v., nhưng trước hết bản chất của dịch là công việc xử lý ngay chính văn bản. Theo Catford (1965), Hartman \& Stork (1972), Bell (1991), Landa (2006), House (2015), dịch là sự thay thế văn bản ở ngôn ngữ nguồn bằng một văn bản tương đương ở ngôn ngữ đích. Quan điểm của trường phái Skopos (Reiß \& Vermeer, 1984; Holz-Mänttäri, 1986) đề cao vai trò đánh giá bản dịch dựa trên phản hồi của độc giả có phần hợp lý, vì độc giả có kiến thức, văn hoá, nhu cầu, nguyện vọng riêng của họ và bản dịch cần thoả mãn nhu cầu của độc giả. Tuy nhiên, giống như bản thân ngôn ngữ, dịch có hai chức năng cơ bản là chức năng ý niệm và chức năng liên nhân (House, 2001). Hai chức năng này tương đương với hai bước khác nhau trong quá trình đánh giá. Bước thứ nhất là phân tích, mô tả, giải thích và so sánh về mặt văn bản và ngôn ngữ (linguistic-textual analysis, description, explanation, comparison) dựa trên nghiên cứu thực nghiệm và kiến thức chuyên môn của người đánh giá. Bước tiếp theo là xét đến những nhận xét mang tính xã hội. Nói cách khác, bước thứ hai mới là đánh giá dựa trên phản hồi của độc giả. Nếu không thực hiện bước thứ nhất, bước thứ hai sẽ không có ý nghĩa. Như vậy, House cho rằng khi đánh giá bản dịch, việc mô tả về ngôn ngữ-văn bản cần được thực hiện trước khi nghiên cứu những vấn đề về tâm lý phức tạp dựa trên trực giác, cảm tính, suy nghĩ của độc giả. Hơn nữa, việc đánh giá bản dịch dựa trên tâm lý, cảm tính, suy nghĩ của độc giả là vấn đề thiếu tính khả thi trong thực tiễn.

\section{Kết luận và đề xuất cho đánh giá dịch Anh-Việt}

Trong bài báo này, chúng tôi đã trình bày về điểm mạnh cũng như hạn chế của một vài hướng tiếp cận chính trong đánh giá dịch thuật, bao gồm tiền ngôn ngữ, đánh giá bản dịch dựa trên phản hồi của độc giả, và đánh giá bản dịch dựa trên phân tích văn bản và diễn ngôn. Để người đánh giá có thể áp dụng một lý thuyết vào thực tiễn đánh giá thì cần có những mô hình với các thao tác cụ thể. Mô hình của House (1977, 1997, 2015) được thừa nhận và áp dụng trong giới học thuật và được tác giả chỉnh sửa, cập nhật trong nhiều năm $(1977,1997,2015)$ được lựa chọn để mô tả và phân tích, từ đó chúng tôi rút ra những đề xuất cho đánh giá dịch Anh-Việt.

Các nghiên cứu trước đây (Hatim \& Mason, 1997; Hewson \& Martin, 1991; Melis \& Hurtado, 2001; Williams \& Chesterman, 2002; Kiraly, 2005; Baker, 2011; Anari \& Ghaffarof, 2013; Drugan, 2013; Colina, 2015) chỉ ra rằng đánh giá dịch thuật phù hợp với ba lĩnh vực của dịch, bao gồm:

(1) đánh giá dịch các bản dịch đã được công bố, xuất bản;

(2) đánh giá bản dịch của các dịch giả chuyên nghiệp;

(3) đánh giá dịch trong môi trường đào tạo dịch.

Có thể thấy, mô hình chức năng dụng học của House (2015), với trọng tâm hướng đến dịch phẩm, có thể áp dụng để đánh giá một bản dịch đã được công bố, xuất bản hoặc một tác phẩm, một dịch phẩm của dịch giả chuyên nghiệp đã được xuất bản. Hơn nữa, mô hình của House (2015) yêu cầu kiến thức chuyên sâu về ngôn ngữ học và các hiểu biết liên quan khác nên nó mang tính hàn lâm cao, cho nên mô hình này còn có thể được sử dụng trong đánh giá chất lượng dịch thuật phục vụ các mục đích nghiên cứu ở các viện nghiên cứu và trường đại học (Lê Hùng Tiến, 2015). 
Ngoài ra, cơ sở lý luận từ mô hình cho thấy khi đánh giá các văn bản ở cặp ngôn ngữ Anh-Việt, việc phân tích các yếu tố nội ngôn và ngoại ngôn của cả bản gốc và bản dịch trong quá trình đánh giá bản dịch là thao tác đóng vai trò quan trọng. Khi áp dụng mô hình chức năng dụng học của House (2015), người đánh giá phân tích văn bản theo các phương tiện từ vựng, cú pháp, phương tiện liên kết văn bản của Trường, Không khí (quan hệ xã hội của những người tham gia giao tiếp, lai lịch của tác giả, v.v), Phương thức và Thể loại văn bản. Việc phân tích văn bản như vậy giúp người đánh giá hiểu được đầy đủ chức năng của văn bản trong tình huống, văn cảnh cụ thể.

Thêm vào đó, mô hình của House (2015) cho thấy tính khả thi đối với đánh giá dịch AnhViệt ở phương diện văn hoá. Theo các nhà ngôn ngữ học trên thế giới và Việt Nam (Austin, 1962; Searle, 1972; Hymes, 1974; Halliday, 1973; Cao Xuân Hạo, 1991; Nguyễn Thiện Giáp, 1999), ngôn ngữ chịu sự chi phối của nhiều yếu tố hoàn cảnh như xã hội, thái độ, giá trị và niềm tin của người tham gia giao tiếp, mối quan hệ giữa những người tham gia giao tiếp. Trong quá trình đánh giá dịch giữa hai nền văn hoá khác nhau, hai ngôn ngữ khác nhau Anh-Việt, sự khác biệt về văn hoá là điều khó tránh khỏi. Chính vì vậy, khía cạnh văn hoá cần được xét tới trong quá trình đánh giá dịch Anh-Việt. Mô hình của House (2015) được nghiên cứu dựa trên khối liệu (corpus studies) để xác định thể loại văn bản và nghiên cứu dựa trên dụng học đối chiếu (contrastive pragmatics) để lọc văn hoá (cultural filtering), đóng góp trong việc giải quyết sự khác biệt về văn hoá.

\section{Tài liệu tham khảo}

\section{Tiếng Việt}

Lê Hoài Ân (2017). Lý thuyết dịch chức năng trường phái Đức và đề xuất hướng thiết kế các bài tập luyện kĩ năng dịch. Kỷ yếu Hội thảo quốc gia Nghiên cúu và giảng dạy ngoại ngũu, ngôn ngũ và quốc tế học tại Việt Nam. Hà Nội, tháng 4, 2017, trang 13-27.

Nguyễn Thiện Giáp (2000). Dụng học Việt ngũ. Hà Nội: NXB Đại học Quốc gia Hà Nội.
Cao Xuân Hạo (1991). Tiếng Việt: So thảo ngũ pháp chúc năng, quyển 1. Hà Nội: NXB Khoa học xã hội.

Lê Hùng Tiến (2015). Mô hình đánh giá dịch thuật tác phẩm văn học Anh-Việt. Kỷ yếu Hội thảo khu vực Nghiên cưu liên ngành về ngôn ngũ học và giảng dạy ngôn ngũu. Huế, tháng 11, 2015, trang 285-297.

Hồ Đắc Túc (2012). Dịch thuật và Tụ do. Công ty TNHH Sách Phương Nam và Đại học Hoa Sen. Hà Nội: NXB Hồng Đức.

\section{Tiếng Anh}

Alikhademi, A. (2015). The application of House's model on Langman's medical embryology and its Persian translation. British Journal of Education, 3(2), 1-20.

Al-Qinai, J. (2000). Translation Quality Assessment. Strategies, Parameters and Procedures. Translators' Journal, 45(3), 497-519.

Austin, J. (1962). How to do things with words. Oxford: Claren Press.

Baker, M. (1992). In other words: A coursebook on translation. London and New York: Routledge.

Bell, R. T. (1991). Translation and Translating: Theory and Practice. London and New York: Routledge.

Brunette, L. (2000). Towards a Terminology for Translation Quality Assessment. The Translator, 6(2), 169-182.

Catford, J, C. (1965). A Linguistic Theory of Translation: an Essay on Applied Linguistics. London: Oxford University Press.

Colina, S. (2008). Translation Quality Evaluation: Empirical Evidence for a Functionalist Approach. The Translator, 14(1), 97-134.

Colina, S. (2015). Fundamentals of translation. Cambridge: Cambridge University Press.

Crystal, D. \& Davy, D. (1969). Investigating English Style. London: Longman.

Derewianka, B. (1990). Exploring How Text Works. Virginia: Primary English Teaching Association.

Drugan, J. (2013). Quality in professional translation: Assessment and improvement. London: Bloomsbury Academic Publishing.

Ehsani, F. \& Zohrabi, K. (2014). Assessing Translation of Advertising text (English-Persian) based on House's Functional-Pragmatic Model of TQA. Procedia-Social and Behavioral Sciences, 98(2014), 420-424. Retrieved September 4, 2017 from https:// doi.org/10.1016/j.sbspro.2014.03.435

Firth, J. R. (1957). Modes of Meanings. In Papers in Linguistics, 1934-51. London: Oxford University Press.

Gutt, E. $\left(1991,2^{\text {nd }}\right)$. Translation and Relevance: Cognition and Context. Oxford: Blackwell; Manchester: St Jerome. 
Halliday, M. A. K. (1973). Explorations in the Functions of Language. London: Arnold.

Halliday, M. A. K., \& Hasan, R. (1989). Language, Context and Text: Aspects of Language in a Social Semiotic Perspective. Oxford: Oxford University Press.

Hartman, R.R.K., \& Stork, F.C. (1972). Dictionary of Language and Linguistics. London: Applied Science.

Hassan, A. E. B., (2015). A translation quality assessment of the English translation of the Hilali Epic. Journal of Arts \& Humanities, 4(12), Retrieved July 10, from http://www.theartsjournal.org/index.php/site/ article/view/890.

Hatim, B., \& Mason, I. (1997). The Translator as Communicator. London \& New: Routledge York.

Hatim, B., \& Munday, J. (2004). Translation: An Advanced Resource Book. USA and Canada: Routledge.

Hewson, L., \& Martin, J. (1991). Redefining translation. The variational approach. London: Routledge.

Hickey, L. (1998). The pragmatics of translation. Clevedon: Multilingual Matters Ltd.

Holz-Mänttäri, J. (1986) Translatorisches Handeln: Theorie und Methode. Helsinki: Suomalainen tiedeakatemia.

House, J. (1977). A Model for Translation Quality Assessment. Tübingen: Gunter Narr.

House, J. (1981). A Model for Translation Quality Assessment. Tübingen: Gunter Narr.

House, J. (1997). Translation Quality Assessment: A Model Revisited. Tübingen: Gunter Narr.

House, J. (2001). Translation Quality Assessment: Linguistic Description versus social evaluation. Meta: Translator's Journal, 46.

House, J. (2015). Translation Quality Assessment. Past and Present. New York: Routledge.

Hymes, D. H. (1964). Language in culture and society: A reader in linguistics and anthropology. New York: Harper \& Row.

Hymes, D. H. (1974). Foundations in sociolinguistics: An ethnographic approach. Philadelphia: University of Pennsylvania Press.

Jiang, C. (2010). Quality assessment for the translation of museum texts application of a systemic functional model. Journal Perspectives: Study in Translation Theory and Practice. 18(2). Retrieved July 15, from http://dx.doi.org/10.1080/09076761003678734

Kargarzadeh, F., \& Paziresh, A. (2017). Assessing the Quality of Persian Translation of Kite Runner based on House's (2014) Functional Pragmatic Model. International Journal of English Language \& Translation Studies. 5(1), 117-126.

Kiraly, D. (2005). A social constructivist approach to translator education: Empowerment from theory to practice. Machester: St. Jerome Publications.

Landa, M. (2006). On Defining Translation. Translator's Journal, 51(3), 435-444.

Lauscher, S. (2000). Translation Quality Assessment: Where can Theory and Practice Meet? The Translator, 6(2), 149-168.
Lyons, J. (1969). Introduction to Theoretical Linguistics. Cambridge: Cambridge University Press.

Malinowski, B. (1923). The Problem of Meaning in Primitive Languages. In The Meaning of Meaning. Ogden, C. K. \& I. A. Richards (Eds.) New York: Harcourt Brace.

Manfredi, M. (2012). Translating text and context: Translation studies and systemic functional linguistics. From theory to practice, Vol 2. Retrieved from July 15, from http://amsacta.unibo.it/3219/

Martin, J. R. \& Rose, D. (2008). Genre Relation: Mapping Culture. Sydney: Equinox.

Melis, M., \& Hurtado, N. Y. A. (2001). Assessment in translation studies: Research needs. Meta: Translators' Journal, 46(2), 272-287.

Moskal, B. M. (2000). Scoring rubrics: what, when and how. Practical Assessment, Research \& Evaluation, 7. Retrieved June 10, 2017 from http://pareonline. net/getvn.asp? $\mathrm{v}=7 \& \mathrm{n}=25$

Mossop, B. (2007). Revising and editing for translators. Manchester: St. Jerome.

Munday, J. (2016). Introducing Translation Studies: Theories and Applications (Eds.). London and New York: Taylor \& Francis.

Munday, J. (2008). Style and Ideology in Translation: Latin American Writing in English. New York: Routledge.

Nazhand, N., \& MohebbiPur, M. (2011). The Application of House's Model to William Faulkner "The Sound and the Fury" and its Persian Translation by Saleh Hosseini. Germany: Verlag, VDM Verlag.

Newmark, P. (1988). A Textbook of Translation. New York: Prentice Hall International.

Nida, E. A. (1964). Towards a Science of Translating. Leiden: E.J. Brill.

Nida, E. A., \& Taber, C. (1969). The Theory and Practice of Translation. Leiden, the Netherlands: E.J. Brill.

Nord, C. (1991/ 2005). Text Analysis in Translation: Theory, Methodology, and Didactic Application of a Model for Translation-oriented Text Analysis. Amsterdam: Rodopi.

Nord, C. (1997). Translating as a Purposeful Activity: Functionalist Approaches Explained. Manchester: St. Jerome.

Phạm Thị Thuỷ (2013). House's functional-pragmatic model of translation assessment and implications for evaluating English-Vietnamese translation quality. VNU Journal of Foreign Studies, 29(1), 56-64.

Rahmanian, H. (2015). The Evaluation of the Quality of Persian Translation of Emily Dickinson's Poems based on the TQA Model of Juliane House. Translation Journal. Retrieved July 25, from http:// translationjournal.net/January-2016/the-evaluationof-the-quality-of-persian-translation-of-emilydickinson-s-poems-based-on-the-tqa-model-ofjuliane-house.html

Reiß, K. \& Vermeer, H. (1984). Translation Theorie. Tübingen: Niemeyer. 
Rhodes, F. E. (2014). Translation criticism: Potentials and Limitations. London and New York: Routledge, Taylor and Francis Group.

Schäffner, C. (1997). From "Good" to "Functionally appropriate": Assessing Translation Quality. Current Issues in Language and Society, 4(1), 1-5.

Searle, J. R. (1972). Speech Acts. Cambridge: Cambridge University Press.

Steiner, E. (1998). A Register-based Translation Evaluation: An Advertisement as a Case in Point. Target 10(2), 291-318.

Tabrizi, H. H., Chalak, A. \& Taheroun, H. A. (2013), Assessing the Quality of Persian Translation of Orwell's Nineteen Eighty-Four Based on House's Model: Overt-Covert Translation Distinction. International Journal of Foreign Language Teaching and Research. 1(2), 1-10.

Teich, E. (2004). Cross-Linguistic Variation in System and Text. Amsterdam: Benjamins.

Toury, G. (1995). Descriptive Translation Studies and Beyond. Amsterdam \& Philadelphia: John Benjamins Publishing.

Venuti, L. (1995). The Translator's Invisibility. A History of Translation. New York, USA and Canada: Routledge.

Waddington, C. (2001). Different Methods of Evaluating Student Translations: The Question of Validity. Meta: Translators' Journal, 46(2), 311325. Retrieved November 10, 2010, from http:// id.erudit.org/iderudit/004583ar

Williams, J., \& Chesterman, A. (2002). The map: A beginner's guide to doing research in translation studies. Manchester, UK: St. Jerome Publishing.

Williams, M. (2009). Translation Quality Assessment. Ottawa: University of Ottawa Press.
Wilss, W. (1996). Knowledge and skills in translator behavior. Amsterdam and Philadelphia: Benjamins.

Zekri, A., \& Shahsavar, Z. (2016). Quality assessment of Persian Translation of English Pharmaceutical Leaflets based on House's model. International Journal of English Language \& Translation Studies, 4(4), 67-76.

Luận văn, luận án chưa công bố

Cao Huyền Trang (2013). A translation quality assessment of chapter 6 in the novel "Lady Chatterley's lover" using J. House's model. Unpublished master thesis. Hanoi: VNU Library and Information Center.

Đặng Thị Phượng (2013). A translation quality assessment of the first three chapters of the novel "Mat ma Da Vinci" by Do Thu Ha (2005) based on House's model. Unpublished master thesis. Hanoi: VNU Library and Information Center.

Lê Mỹ Hạnh (2009). A translation quality assessment of the Vietnamese version of part 7 "Nightmare" in the Novel using J. House's model. Unpublished master thesis. Hanoi: VNU Library and Information Center.

Phạm Thị Thuỷ (2015). An evaluation of translation quality of literary works (prose). Unpublished doctoral thesis. Hanoi: VNU Library and Information Center.

Triệu Thu Hằng (2015). A translation quality assessment on Vietnamese translation of "The Great Gatsby" by F. Scott Fotzgerald. Unpublished master thesis. Hanoi: VNU Library and Information Center.

\title{
TRANSLATION QUALITY ASSESSMENT MODELS AND IMPLICATIONS FOR ENGLISH-VIETNAMESE TRANSLATION QUALITY ASSESSMENT
}

\author{
Trieu Thu Hang \\ Faculty of English Language Teacher Education, VNU University of Languages and International \\ Studies, Pham Van Dong, Cau Giay, Hanoi, Vietnam
}

\begin{abstract}
The paper firstly aims to review different schools of thought in translation quality assessment (TQA), namely mentalist views; response-based approach; and text and discoursebased approach. As a representative model of text and discourse-based approach, House's model (2015) is described, analyzed and discussed for the practice of translation assessment. Based on the theoretical discussion on House's model (2015), the implications for English-Vietnamese translation assessment are finally drawn out.

Keywords: translation quality assessment, response-based approach, text and discourse-based approach, House's model (2015)
\end{abstract}

